MATHEMATICS OF COMPUTATION

Volume 71, Number 238 , Pages $537-552$

S 0025-5718(01)01335-7

Article electronically published on September 17, 2001

\title{
ANALYSIS OF A FINITE ELEMENT METHOD FOR PRESSURE/POTENTIAL FORMULATION OF ELASTOACOUSTIC SPECTRAL PROBLEMS
}

\author{
ALFREDO BERMÚDEZ AND RODOLFO RODRÍGUEZ
}

\begin{abstract}
A finite element method to approximate the vibration modes of a structure enclosing an acoustic fluid is analyzed. The fluid is described by using simultaneously pressure and displacement potential variables, whereas displacement variables are used for the solid. A mathematical analysis of the continuous spectral problem is given.

The problem is discretized on a simplicial mesh by using piecewise constant elements for the pressure and continuous piecewise linear finite elements for the other fields. Error estimates are settled for approximate eigenvalues and eigenfrequencies. Finally, implementation issues are discussed.
\end{abstract}

\section{INTRODUCTION}

In this paper we analyze a finite element method for the numerical solution of a spectral problem arising in fluid-solid interactions. It concerns the numerical computation of internal elastoacoustic vibrations, i.e., harmonic vibrations of a coupled system consisting of an elastic solid enclosing an acoustic (compressible, inviscid and barotropic) fluid.

A first possibility to solve this problem is to consider a formulation in terms of displacements in the solid and pressure in the fluid (see [17]). However, such an approach leads to nonsymmetric eigenvalue problems, which is an inconvenient from the numerical point of view.

An alternative procedure has been recently introduced in [5] (see also [2, 3. 4). It is based on using displacement variables also for the fluid, discretized by lowest degree Raviart-Thomas finite elements on a triangular (or tetrahedral) mesh. Interface coupling between this discretization and classical piecewise linear finite elements for the solid displacements is achieved in a nonconforming way. Error estimates have been obtained and it has been proved that no spurious modes arise as is typical in other discretizations of this formulation (see [10]).

Another approach, also leading to symmetrical spectral problems, has been introduced in [13. It consists of using simultaneously the pressure and the potential of displacements to describe the fluid motion. In the present paper we analyze a finite

Received by the editor April 13, 1999 and, in revised form, August 14, 2000.

2000 Mathematics Subject Classification. Primary 65N25, 65N30; Secondary 70J30, 74F10, $76 Q 05$.

Key words and phrases. Finite element spectral approximation, elastoacoustic vibrations.

The first author was supported by DGESIC project PB97-0508 (Spain).

The second author was supported by FONDECYT No. 1.990.346 and FONDAP in Applied Mathematics (Chile). 
element method for the numerical solution of this pressure/potential formulation: standard continuous piecewise linear finite elements are used for the solid displacements and the potential variables, combined with piecewise constant elements for the pressure. We show that this discretization leads to a sparse symmetric eigenvalue problem involving only scalar variables for the fluid. This analysis is valid for both two- and three-dimensional problems.

Numerical results of the application of this method to some test examples have been reported in [12, 13]. In the first of these references it is also shown that the pressure variables can be further eliminated, to reduce the number of degrees of freedom, in an inner step of a "shift and invert" eigensolver without destroying the sparseness of the involved matrices.

We start by giving a weak formulation of the spectral problem which allows us to characterize its solutions and to state their regularity properties. Then we introduce the discretization and prove error estimates both for eigenfunctions and eigenvalues. Finally, an alternative formulation of the discrete spectral problem, more convenient from the computational point of view, is proved to be equivalent to the one theoretically analyzed.

\section{THE MODEL PROBLEM}

We consider the problem of determining the vibration modes of a linear elastic structure containing a compressible, inviscid and barotropic fluid. Our model problem consists of a vessel completely filled with fluid, as shown in Figure 1, In spite of the fact that this figure is two-dimensional, all the subsequent analysis is valid for $3 \mathrm{D}$ as well as for $2 \mathrm{D}$ problems. However, for the sake of definiteness, we will use 3D terminology if necessary.

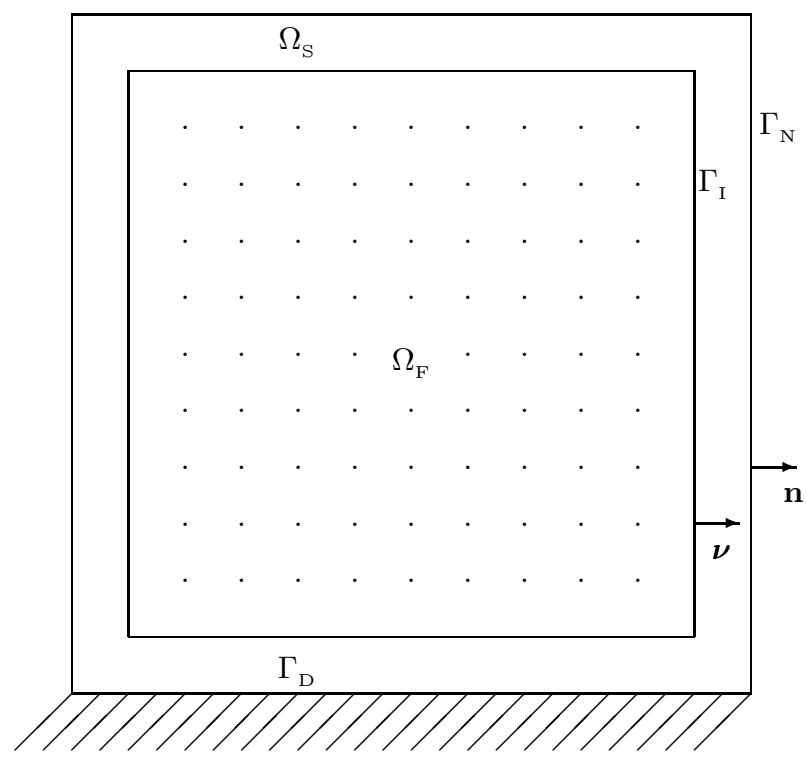

Figure 1. Fluid and solid domains. 
Let $\Omega_{\mathrm{F}}$ and $\Omega_{\mathrm{S}}$ be the polyhedral domains in $\mathbb{R}^{n}(n=2$ or 3 ) occupied by the fluid and the solid, respectively; they are not supposed to be either convex or simply connected. Let us denote by $\Gamma_{\mathrm{I}}$ the interface between solid and fluid and by $\boldsymbol{\nu}$ its unit normal vector pointing outwards from $\Omega_{\mathrm{F}}$. The external solid boundary is assumed to be the union of two polyhedral surfaces: $\Gamma_{\mathrm{D}}$ and $\Gamma_{\mathrm{N}}$; the structure is supposed to be free along $\Gamma_{\mathrm{N}}$ and fixed along $\Gamma_{\mathrm{D}}$ (for simplicity, meas $\left(\Gamma_{\mathrm{D}}\right)>0$ is also assumed). Finally, $\mathbf{n}$ denotes the unit outward normal vector along $\Gamma_{\mathrm{N}}$.

Throughout this paper we will use standard notation for Sobolev spaces and norms. Furthermore, we will denote by $C$ a generic constant not necessarily the same at each occurrence.

The physical magnitudes of the fluid will be denoted by

- $\mathbf{u}$ : the displacement vector field,

- $p$ : the pressure,

- $c$ : the sound speed,

- $\rho_{\mathrm{F}}$ : the density;

and those of the solid by

- v: the displacement vector field,

- $\rho_{\mathrm{S}}$ : the density,

- $\lambda_{\mathrm{S}}$ and $\mu_{\mathrm{S}}$ : the Lamé coefficients,

- $\varepsilon(\mathbf{v})$ : the strain tensor defined by

$$
\varepsilon_{i j}(\mathbf{v}):=\frac{1}{2}\left(\frac{\partial v_{i}}{\partial x_{j}}+\frac{\partial v_{j}}{\partial x_{i}}\right), \quad i, j=1, \ldots, n,
$$

- $\boldsymbol{\sigma}(\mathbf{v})$ : the stress tensor, which we assume to be related to the strains by Hooke's law:

$$
\sigma_{i j}(\mathbf{v})=\lambda_{\mathrm{S}} \sum_{k=1}^{n} \varepsilon_{k k}(\mathbf{v}) \delta_{i j}+2 \mu_{\mathrm{s}} \varepsilon_{i j}(\mathbf{v}), \quad i, j=1, \ldots, n .
$$

We are interested in the small amplitude motions departing from the rest. The classical linearization procedure yields the following eigenvalue problem for the free vibration modes of the coupled system and their corresponding frequencies $\omega$ (see, for instance, [14]):

Find $\omega>0, \mathbf{u}: \Omega_{\mathrm{F}} \longrightarrow \mathbb{R}^{n}, \mathbf{v}: \Omega_{\mathrm{S}} \longrightarrow \mathbb{R}^{n}$ and $p: \Omega_{\mathrm{F}} \longrightarrow \mathbb{R},(\mathbf{u}, \mathbf{v}, p) \neq \mathbf{0}$, such that

$$
\begin{aligned}
\boldsymbol{\nabla} p-\omega^{2} \rho_{\mathrm{F}} \mathbf{u}=\mathbf{0} & \text { in } \Omega_{\mathrm{F}}, \\
p+\rho_{\mathrm{F}} c^{2} \operatorname{div} \mathbf{u}=0 & \text { in } \Omega_{\mathrm{F}}, \\
\operatorname{div}[\boldsymbol{\sigma}(\mathbf{v})]+\omega^{2} \rho_{\mathrm{S}} \mathbf{v}=\mathbf{0} & \text { in } \Omega_{\mathrm{S}}, \\
\mathbf{u} \cdot \boldsymbol{\nu}=\mathbf{v} \cdot \boldsymbol{\nu} & \text { on } \Gamma_{\mathrm{I}}, \\
\boldsymbol{\sigma}(\mathbf{v}) \boldsymbol{\nu}+p \boldsymbol{\nu}=\mathbf{0} & \text { on } \Gamma_{\mathrm{I}}, \\
\boldsymbol{\sigma}(\mathbf{v}) \mathbf{n}=\mathbf{0} & \text { on } \Gamma_{\mathrm{N}}, \\
\mathbf{v}=\mathbf{0} & \text { on } \Gamma_{\mathrm{D}} .
\end{aligned}
$$

Let us introduce a new variable, $\phi:=\frac{1}{\rho_{\mathrm{F}} \omega^{2}} p$, for the potential of the fluid displacement field (indeed, from (2.1) we deduce $\mathbf{u}=\boldsymbol{\nabla} \phi$ in $\Omega_{\mathrm{F}}$ ). Then the fluid displacement $\mathbf{u}$ can be eliminated from (2.1), (2.2) and (2.4). Furthermore, we can 
replace $p$ in (2.5) by $\rho_{\mathrm{F}} \omega^{2} \phi$ to obtain the following set of equations equivalent to (2.1)-(2.7):

$$
\begin{aligned}
\rho_{\mathrm{F}} \Delta \phi+\frac{1}{c^{2}} p=0 & \text { in } \Omega_{\mathrm{F}}, \\
p=\rho_{\mathrm{F}} \omega^{2} \phi & \text { in } \Omega_{\mathrm{F}}, \\
\operatorname{div}[\boldsymbol{\sigma}(\mathbf{v})]+\omega^{2} \rho_{\mathrm{S}} \mathbf{v}=\mathbf{0} & \text { in } \Omega_{\mathrm{S}}, \\
\frac{\partial \phi}{\partial \boldsymbol{\nu}}=\mathbf{v} \cdot \boldsymbol{\nu} & \text { on } \Gamma_{\mathrm{I}}, \\
\boldsymbol{\sigma}(\mathbf{v}) \boldsymbol{\nu}+\rho_{\mathrm{F}} \omega^{2} \phi \boldsymbol{\nu}=\mathbf{0} & \text { on } \Gamma_{\mathrm{I}} . \\
\boldsymbol{\sigma}(\mathbf{v}) \mathbf{n}=\mathbf{0} & \text { on } \Gamma_{\mathrm{N}}, \\
\mathbf{v}=\mathbf{0} & \text { on } \Gamma_{\mathrm{D}} .
\end{aligned}
$$

Let $\mathcal{X}$ denote the product space defined by

$$
\mathcal{X}:=H_{\Gamma_{\mathrm{D}}}^{1}\left(\Omega_{\mathrm{S}}\right)^{n} \times L^{2}\left(\Omega_{\mathrm{F}}\right),
$$

where $H_{\Gamma_{\mathrm{D}}}^{1}\left(\Omega_{\mathrm{S}}\right)^{n}:=\left\{\mathbf{w} \in H^{1}\left(\Omega_{\mathrm{S}}\right)^{n}:\left.\mathbf{w}\right|_{\Gamma_{\mathrm{D}}}=\mathbf{0}\right\}$. We endow $\mathcal{X}$ with the usual product norm

$$
\|(\mathbf{w}, q)\|:=\left[\|\mathbf{w}\|_{H^{1}\left(\Omega_{\mathrm{S}}\right)^{n}}^{2}+\|q\|_{L^{2}\left(\Omega_{\mathrm{F}}\right)}^{2}\right]^{1 / 2} .
$$

To give a weak formulation of the spectral problem above, we first multiply (2.10) by a test function $\mathbf{w} \in H_{\Gamma_{\mathrm{D}}}^{1}\left(\Omega_{\mathrm{S}}\right)^{n}$ and then integrate in $\Omega_{\mathrm{S}}$. By using a Green's formula and taking into account (2.13) and (2.12) we obtain

$$
\int_{\Omega_{\mathrm{S}}} \boldsymbol{\sigma}(\mathbf{v}): \varepsilon(\mathbf{w})-\omega^{2} \int_{\Gamma_{\mathrm{I}}} \rho_{\mathrm{F}} \phi \mathbf{w} \cdot \boldsymbol{\nu} \mathrm{d} \Gamma=\omega^{2} \int_{\Omega_{\mathrm{S}}} \rho_{\mathrm{S}} \mathbf{v} \cdot \mathbf{w}
$$

Next, we multiply (2.9) by $1 / \rho_{\mathrm{F}} c^{2}$ times a test function $q \in L^{2}\left(\Omega_{\mathrm{F}}\right)$, integrate in $\Omega_{\mathrm{F}}$ and add the resulting equation to (2.15). We get

$$
\begin{aligned}
\int_{\Omega_{\mathrm{S}}} \boldsymbol{\sigma}(\mathbf{v}): \varepsilon(\mathbf{w})+\int_{\Omega_{\mathrm{F}}} \frac{1}{\rho_{\mathrm{F}} c^{2}} p q \\
\quad=\omega^{2}\left(\int_{\Omega_{\mathrm{S}}} \rho_{\mathrm{S}} \mathbf{v} \cdot \mathbf{w}+\int_{\Omega_{\mathrm{F}}} \frac{1}{c^{2}} \phi q+\int_{\Gamma_{\mathrm{I}}} \rho_{\mathrm{F}} \phi \mathbf{w} \cdot \boldsymbol{\nu} \mathrm{d} \Gamma\right) .
\end{aligned}
$$

Similarly, by multiplying (2.8) by a test function $\psi \in H^{1}\left(\Omega_{\mathrm{F}}\right)$, integrating in $\Omega_{\mathrm{F}}$ and using a Green's formula and (2.11), we obtain

$$
\int_{\Omega_{\mathrm{F}}} \rho_{\mathrm{F}} \boldsymbol{\nabla} \phi \cdot \nabla \psi=\int_{\Omega_{\mathrm{F}}} \frac{1}{c^{2}} p \psi+\int_{\Gamma_{\mathrm{I}}} \rho_{\mathrm{F}} \mathbf{v} \cdot \boldsymbol{\nu} \psi \mathrm{d} \Gamma .
$$

Notice that this equation makes sense only if the following compatibility constraint holds:

$$
\int_{\Omega_{\mathrm{F}}} \frac{1}{c^{2}} p+\int_{\Gamma_{\mathrm{I}}} \rho_{\mathrm{F}} \mathbf{v} \cdot \boldsymbol{\nu} \mathrm{d} \Gamma=0
$$

In that case, the potential $\phi$ is uniquely determined up to an additive constant by equation (2.17). Furthermore, if the test functions in (2.16), $\mathbf{w} \in H_{\Gamma_{\mathrm{D}}}^{1}\left(\Omega_{\mathrm{S}}\right)^{n}$ and $q \in L^{2}\left(\Omega_{\mathrm{F}}\right)$, are chosen so as to satisfy also this constraint, (2.16) will be valid independently of this additive constant. 
Let us denote by $\mathcal{V}$ the closed subspace of $\mathcal{X}$ given by

$$
\mathcal{V}:=\left\{(\mathbf{w}, q) \in \mathcal{X}: \int_{\Omega_{\mathrm{F}}} \frac{1}{c^{2}} q+\int_{\Gamma_{\mathrm{I}}} \rho_{\mathrm{F}} \mathbf{w} \cdot \boldsymbol{\nu} \mathrm{d} \Gamma=0\right\}
$$

and let $\mathcal{W}:=H^{1}\left(\Omega_{\mathrm{F}}\right) / \mathbb{R}$, endowed with the standard quotient space norm. Thus, (2.16) and 2.17) show that any solution of the original spectral problem 2.1 - 2.7) also satisfies the following weak formulation:

Find $\omega \geq 0$ and $\mathbf{0} \neq(\mathbf{v}, p) \in \mathcal{V}$ such that

$$
\begin{aligned}
& \int_{\Omega_{\mathrm{S}}} \boldsymbol{\sigma}(\mathbf{v}): \boldsymbol{\varepsilon}(\mathbf{w})+\int_{\Omega_{\mathrm{F}}} \frac{1}{\rho_{\mathrm{F}} c^{2}} p q \\
& \quad=\omega^{2}\left(\int_{\Omega_{\mathrm{S}}} \rho_{\mathrm{S}} \mathbf{v} \cdot \mathbf{w}+\int_{\Omega_{\mathrm{F}}} \frac{1}{c^{2}} \phi q+\int_{\Gamma_{\mathrm{I}}} \rho_{\mathrm{F}} \phi \mathbf{w} \cdot \boldsymbol{\nu} \mathrm{d} \Gamma\right) \quad \forall(\mathbf{w}, q) \in \mathcal{V},
\end{aligned}
$$

with $\phi \in \mathcal{W}$ such that

$$
\int_{\Omega_{\mathrm{F}}} \rho_{\mathrm{F}} \boldsymbol{\nabla} \phi \cdot \nabla \psi=\int_{\Omega_{\mathrm{F}}} \frac{1}{c^{2}} p \psi+\int_{\Gamma_{\mathrm{I}}} \rho_{\mathrm{F}} \mathbf{v} \cdot \boldsymbol{\nu} \psi \mathrm{d} \Gamma \quad \forall \psi \in \mathcal{W} .
$$

In the following section we will prove further regularity of the eigenfunctions of this spectral problem. As a by-product, we will show that all the solutions of (2.18)-2.19) also solve (2.1)-(2.7) with $\mathbf{u}=\boldsymbol{\nabla} \phi$.

For theoretical purposes it will be useful to consider also the variational spectral problem obtained by eliminating $\phi$ in (2.18)-2.19). Let us denote by $\mathcal{M}$ the bounded linear operator

$$
\mathcal{M}: \begin{gathered}
\mathcal{V} \\
(\mathbf{f}, g)
\end{gathered}
$$

with $\phi$ being the unique solution in $\mathcal{W}$ of the compatible Neumann problem

$$
\int_{\Omega_{\mathrm{F}}} \rho_{\mathrm{F}} \boldsymbol{\nabla} \phi \cdot \nabla \psi=\int_{\Omega_{\mathrm{F}}} \frac{1}{c^{2}} g \psi+\int_{\Gamma_{\mathrm{I}}} \rho_{\mathrm{F}} \mathbf{f} \cdot \boldsymbol{\nu} \psi \mathrm{d} \Gamma \quad \forall \psi \in \mathcal{W} .
$$

Therefore problem (2.18)-2.19) is equivalent to the following one:

Find $\omega \geq 0$ and $\mathbf{0} \neq(\mathbf{v}, p) \in \mathcal{V}$ such that:

$$
\begin{array}{r}
\int_{\Omega_{\mathrm{S}}} \boldsymbol{\sigma}(\mathbf{v}): \boldsymbol{\varepsilon}(\mathbf{w})+\int_{\Omega_{\mathrm{F}}} \frac{1}{\rho_{\mathrm{F}} c^{2}} p q \\
=\omega^{2}\left[\int_{\Omega_{\mathrm{S}}} \rho_{\mathrm{S}} \mathbf{v} \cdot \mathbf{w}+\int_{\Omega_{\mathrm{F}}} \frac{1}{c^{2}} \mathcal{M}(\mathbf{v}, p) q+\int_{\Gamma_{\mathrm{I}}} \rho_{\mathrm{F}} \mathcal{M}(\mathbf{v}, p) \mathbf{w} \cdot \boldsymbol{\nu} \mathrm{d} \Gamma\right] \\
\forall(\mathbf{w}, q) \in \mathcal{V} .
\end{array}
$$

\section{Characterizhtion of the spectrum and a priori estimates}

Let us now consider the following continuous bilinear forms from $\mathcal{V} \times \mathcal{V} \longrightarrow \mathbb{R}$ :

$$
\begin{aligned}
& a((\mathbf{v}, p),(\mathbf{w}, q)):=\int_{\Omega_{\mathrm{S}}} \boldsymbol{\sigma}(\mathbf{v}): \boldsymbol{\varepsilon}(\mathbf{w})+\int_{\Omega_{\mathrm{F}}} \frac{1}{\rho_{\mathrm{F}} c^{2}} p q, \\
& b((\mathbf{v}, p),(\mathbf{w}, q)):=\int_{\Omega_{\mathrm{S}}} \rho_{\mathrm{S}} \mathbf{v} \cdot \mathbf{w}+\int_{\Omega_{\mathrm{F}}} \frac{1}{c^{2}} \mathcal{M}(\mathbf{v}, p) q+\int_{\Gamma_{\mathrm{I}}} \rho_{\mathrm{F}} \mathcal{M}(\mathbf{v}, p) \mathbf{w} \cdot \boldsymbol{\nu} \mathrm{d} \Gamma .
\end{aligned}
$$

The first one is symmetric and, by Korn's inequality, elliptic on $\mathcal{X}$, and hence on $\mathcal{V}$. Regarding the second one we have the following result: 
Lemma 3.1. The bilinear form $b$ is symmetric and positive definite.

Proof. Let $(\mathbf{v}, p),(\mathbf{w}, q) \in \mathcal{V}$ and $\phi:=\mathcal{M}(\mathbf{v}, p), \psi:=\mathcal{M}(\mathbf{w}, q) \in \mathcal{W}$. Then

$$
\begin{aligned}
b((\mathbf{v}, p),(\mathbf{w}, q)) & =\int_{\Omega_{\mathrm{S}}} \rho_{\mathrm{S}} \mathbf{v} \cdot \mathbf{w}+\int_{\Omega_{\mathrm{F}}} \frac{1}{c^{2}} \phi q+\int_{\Gamma_{\mathrm{I}}} \rho_{\mathrm{F}} \phi \mathbf{w} \cdot \boldsymbol{\nu} \mathrm{d} \Gamma \\
& =\int_{\Omega_{\mathrm{S}}} \rho_{\mathrm{S}} \mathbf{v} \cdot \mathbf{w}+\int_{\Gamma_{\mathrm{I}}} \rho_{\mathrm{F}} \boldsymbol{\nabla} \psi \cdot \nabla \phi \\
& =\int_{\Omega_{\mathrm{S}}} \rho_{\mathrm{S}} \mathbf{v} \cdot \mathbf{w}+\int_{\Omega_{\mathrm{F}}} \frac{1}{c^{2}} p \psi+\int_{\Gamma_{\mathrm{I}}} \rho_{\mathrm{F}} \mathbf{v} \cdot \boldsymbol{\nu} \psi \mathrm{d} \Gamma \\
& =b((\mathbf{w}, q),(\mathbf{v}, p)) .
\end{aligned}
$$

Furthermore,

$$
b((\mathbf{v}, p),(\mathbf{v}, p))=\int_{\Omega_{\mathrm{S}}} \rho_{\mathrm{S}}|\mathbf{v}|^{2}+\int_{\Gamma_{\mathrm{I}}} \rho_{\mathrm{F}}|\nabla \phi|^{2} \geq 0
$$

and this vanishes only if $\mathbf{v} \equiv \mathbf{0}$ and $\boldsymbol{\nabla} \phi \equiv \mathbf{0}$. In this case, by the definition of $\mathcal{M}$, $\int_{\Omega_{\mathrm{F}}} \frac{1}{c^{2}} p \psi=\int_{\Omega_{\mathrm{F}}} \rho_{\mathrm{F}} \boldsymbol{\nabla} \phi \cdot \boldsymbol{\nabla} \psi-\int_{\Gamma_{\mathrm{I}}} \rho_{\mathrm{F}} \mathbf{v} \cdot \boldsymbol{\nu} \psi \mathrm{d} \Gamma=0$ for all $\psi \in \mathcal{W}$. On the other hand, since $(\mathbf{v}, p) \in \mathcal{V}$, then $\int_{\Omega_{\mathrm{F}}} \frac{1}{c^{2}} p=-\int_{\Gamma_{\mathrm{I}}} \rho_{\mathrm{F}} \mathbf{v} \cdot \boldsymbol{\nu} \mathrm{d} \Gamma=0$. Thus $\int_{\Omega_{\mathrm{F}}} \frac{1}{c^{2}} p \psi=0$ for all $\psi \in H^{1}\left(\Omega_{\mathrm{F}}\right)$, and hence $p \equiv 0$.

The previous lemma shows that $b(\cdot, \cdot)$ is an inner product on $\mathcal{V}$. Hence it defines a norm on this space that we denote $|\cdot|$. Furthermore, because of (3.1), the following characterization holds:

$$
|(\mathbf{f}, g)|^{2}:=b((\mathbf{f}, g),(\mathbf{f}, g))=\int_{\Omega_{\mathrm{S}}} \rho_{\mathrm{S}}|\mathbf{f}|^{2}+\int_{\Omega_{\mathrm{F}}} \rho_{\mathrm{F}}|\nabla \phi|^{2}, \quad \text { with } \phi=\mathcal{M}(\mathbf{f}, g) .
$$

In order to analyze our spectral problem we introduce the following bounded linear operator:

$$
\mathbf{T}: \begin{array}{cl}
\mathcal{V} & \longrightarrow \mathcal{V} \\
(\mathbf{f}, g) & \longmapsto(\mathbf{v}, p)
\end{array}
$$

with $(\mathbf{v}, p) \in \mathcal{V}$ being the solution of the elliptic problem

$$
a((\mathbf{v}, p),(\mathbf{w}, q))=b((\mathbf{f}, g),(\mathbf{w}, q)) \quad \forall(\mathbf{w}, q) \in \mathcal{V} .
$$

Notice that the ellipticity of $a$ and the continuity of $b$ yield $\|(\mathbf{v}, p)\| \leq C|(\mathbf{f}, g)|$.

It is simple to show that $\mathbf{T}$ is self-adjoint and positive definite with respect to $a$ and $b$. Hence all of its eigenvalues are real and positive. On the other hand, $(\lambda,(\mathbf{v}, p))$ is an eigenpair of $\mathbf{T}$ if and only if $\omega=\frac{1}{\sqrt{\lambda}}$ and $(\mathbf{v}, p)$ are solutions of (2.21). Therefore, the knowledge of the spectrum of $\mathbf{T}$ gives complete information about the solutions of our original problem.

We have the following a priori estimates for $\mathbf{T}(\mathcal{V})$ :

Lemma 3.2. There exist constants $t \in(0,1]$ and $C>0$ such that if $(\mathbf{v}, p)=\mathbf{T}(\mathbf{f}, g)$ with $(\mathbf{f}, g) \in \mathcal{V}$, then $\mathbf{v} \in H^{1+t}\left(\Omega_{\mathrm{S}}\right)^{n}, p \in H^{1}\left(\Omega_{\mathrm{F}}\right)$ and

$$
\|\mathbf{v}\|_{H^{1+t}\left(\Omega_{\mathrm{S}}\right)^{n}}+\|p\|_{H^{1}\left(\Omega_{\mathrm{F}}\right)} \leq C|(\mathbf{f}, g)| .
$$


Proof. Since $(\mathbf{v}, p)=\mathbf{T}(\mathbf{f}, g)$, then we have

$$
\begin{aligned}
\int_{\Omega_{\mathrm{S}}} \boldsymbol{\sigma}(\mathbf{v}): \boldsymbol{\varepsilon}(\mathbf{w})+\int_{\Omega_{\mathrm{F}}} \frac{1}{\rho_{\mathrm{F}} c^{2}} p q \\
\quad=\int_{\Omega_{\mathrm{S}}} \rho_{\mathrm{S}} \mathbf{f} \cdot \mathbf{w}+\int_{\Omega_{\mathrm{F}}} \frac{1}{c^{2}} \phi q+\int_{\Gamma_{\mathrm{I}}} \rho_{\mathrm{F}} \phi \mathbf{w} \cdot \boldsymbol{\nu} \mathrm{d} \Gamma \quad \forall(\mathbf{w}, q) \in \mathcal{V},
\end{aligned}
$$

for any $\phi \in H^{1}\left(\Omega_{\mathrm{F}}\right)$ such that

$$
\int_{\Omega_{\mathrm{F}}} \rho_{\mathrm{F}} \boldsymbol{\nabla} \phi \cdot \nabla \psi=\int_{\Omega_{\mathrm{F}}} \frac{1}{c^{2}} g \psi+\int_{\Gamma_{\mathrm{I}}} \rho_{\mathrm{F}} \mathbf{f} \cdot \boldsymbol{\nu} \psi \mathrm{d} \Gamma \quad \forall \psi \in \mathcal{W} .
$$

If $\phi$ is chosen as the particular solution of (3.6) satisfying

$$
\int_{\Omega_{\mathrm{F}}} \frac{1}{\rho_{\mathrm{F}} c^{2}} p=\int_{\Omega_{\mathrm{F}}} \frac{1}{c^{2}} \phi
$$

then 3.5 is also true for $(\mathbf{w}, q)=(\mathbf{0}, 1)$, and hence for all $(\mathbf{w}, q) \in \mathcal{X}=\mathcal{V} \oplus$ $\langle\{(\mathbf{0}, 1)\}\rangle$.

In particular, for any $q \in L^{2}\left(\Omega_{\mathrm{F}}\right)$, we may apply 3.5 to $(\mathbf{0}, q) \in \mathcal{X}$ to obtain

$$
\int_{\Omega_{\mathrm{F}}} \frac{1}{\rho_{\mathrm{F}} c^{2}} p q=\int_{\Omega_{\mathrm{F}}} \frac{1}{c^{2}} \phi q \text {. }
$$

Thus $p=\rho_{\mathrm{F}} \phi$ and hence $p \in H^{1}\left(\Omega_{\mathrm{F}}\right)$ with

$$
\|p\|_{H^{1}\left(\Omega_{\mathrm{F}}\right)} \leq C\left[\|p\|_{L^{2}\left(\Omega_{\mathrm{F}}\right)}+\|\boldsymbol{\nabla} \phi\|_{L^{2}\left(\Omega_{\mathrm{F}}\right)^{n}}\right] \leq C|(\mathbf{f}, g)|,
$$

the latter inequality because of $\|(\mathbf{v}, p)\| \leq C|(\mathbf{f}, g)|$ and (3.2).

On the other hand, by testing (3.5) with different $(\mathbf{w}, 0) \in \mathcal{X}$ and using $p=\rho_{\mathrm{F}} \phi$, we have that $\mathbf{v}$ is a solution (in the sense of distributions) of the following elasticity problem:

$$
\begin{aligned}
-\operatorname{div}[\boldsymbol{\sigma}(\mathbf{v})]=\rho_{\mathrm{S}} \mathbf{f} & \text { in } \Omega_{\mathrm{S}}, \\
\boldsymbol{\sigma}(\mathbf{v}) \boldsymbol{\nu}=-p \boldsymbol{\nu} & \text { on } \Gamma_{\mathrm{I}} \\
\boldsymbol{\sigma}(\mathbf{v}) \mathbf{n}=\mathbf{0} & \text { on } \Gamma_{\mathrm{N}} \\
\mathbf{v}=\mathbf{0} & \text { on } \Gamma_{\mathrm{D}} .
\end{aligned}
$$

Therefore, it follows from the results in [9] (see, for instance, [15]) that there exists $t \in(0,1]$ such that $\mathbf{v} \in H^{1+t}\left(\Omega_{\mathrm{S}}\right)^{n}$ with

$$
\|\mathbf{v}\|_{H^{1+t}\left(\Omega_{\mathrm{S}}\right)^{n}} \leq C\left[\|\mathbf{f}\|_{L^{2}\left(\Omega_{\mathrm{S}}\right)^{n}}+\|p\|_{H^{1 / 2}\left(\Gamma_{\mathrm{I}}\right)}\right] \leq C|(\mathbf{f}, g)| .
$$

This lemma allows us to characterize the spectrum of the operator $\mathbf{T}$ :

Theorem 3.3. The spectrum of $\mathbf{T}$ consists of 0 and a sequence of strictly positive finite multiplicity eigenvalues $\left\{\lambda_{k}: k \in \mathbb{N}\right\}$ converging to 0 .

Proof. It is an immediate consequence of Lemma 3.2, the compactness of the inclusion $\left[H^{1+t}\left(\Omega_{\mathrm{S}}\right)^{n} \times H^{1}\left(\Omega_{\mathrm{F}}\right)\right] \cap \mathcal{V} \hookrightarrow \mathcal{V}$ (for $t>0$ ) and the self-adjointness and positive definiteness of $\mathbf{T}$.

The following lemma states a priori estimates for $\mathcal{M}(\mathcal{V})$ : 
Lemma 3.4. There exist constants $s \in\left(\frac{1}{2}, 1\right]$ and $C>0$ such that, if $\phi=\mathcal{M}(\mathbf{f}, g)$ with $(\mathbf{f}, g) \in \mathcal{V}$, then $\phi \in H^{1+s}\left(\Omega_{\mathrm{F}}\right) / \mathbb{R}$ and

$$
\|\boldsymbol{\nabla} \phi\|_{H^{s}\left(\Omega_{\mathrm{F}}\right)^{n}} \leq C\|(\mathbf{f}, g)\| .
$$

Proof. By the definition of $\mathcal{M}, \phi$ is solution of the compatible Neumann problem (2.20); thus

$$
\begin{array}{rr}
-\Delta \phi=\frac{1}{\rho_{\mathrm{F}} c^{2}} g & \text { in } \Omega_{\mathrm{F}}, \\
\frac{\partial \phi}{\partial \boldsymbol{\nu}}=\mathbf{f} \cdot \boldsymbol{\nu} & \text { on } \Gamma_{\mathrm{I}} .
\end{array}
$$

This problem can be transformed into a homogeneous Neumann problem by using the results in [8] on lifting of traces in a polyhedral domain. Then, we can apply the results in [16] to obtain the claimed result.

Now we are able to prove further regularity for the eigenfunctions of our problem, which will be used to obtain error estimates for the finite element method to be introduced in the next section:

Lemma 3.5. Let $(\mathbf{f}, g)$ be an eigenfunction of $\mathbf{T}$. Then $\mathbf{f} \in H^{1+t}\left(\Omega_{\mathrm{S}}\right)^{n}, g \in$ $H^{1+s}\left(\Omega_{\mathrm{F}}\right)$ and

$$
\|\mathbf{f}\|_{H^{1+t}\left(\Omega_{\mathrm{S}}\right)^{n}}+\|g\|_{H^{1+s}\left(\Omega_{\mathrm{F}}\right)} \leq C|(\mathbf{f}, g)|,
$$

with $t \in(0,1]$ and $s \in\left(\frac{1}{2}, 1\right]$ as in Lemmas 3.2 and 3.4 respectively.

Proof. Let $(\mathbf{v}, p)=\mathbf{T}(\mathbf{f}, g)=\lambda(\mathbf{f}, g)$. Since $\lambda>0$, Lemma 3.2 implies

$$
g=\frac{1}{\lambda} p \in H^{1}\left(\Omega_{\mathrm{F}}\right), \quad \text { with } \quad\|g\|_{H^{1}\left(\Omega_{\mathrm{F}}\right)} \leq C|(\mathbf{f}, g)|,
$$

and

$$
\mathbf{f}=\frac{1}{\lambda} \mathbf{v} \in H^{1+t}\left(\Omega_{\mathrm{S}}\right)^{n}, \quad \text { with } \quad\|\mathbf{f}\|_{H^{1+t}\left(\Omega_{\mathrm{S}}\right)^{n}} \leq C|(\mathbf{f}, g)| .
$$

Now, let $\phi \in H^{1}\left(\Omega_{\mathrm{F}}\right)$ be defined by (3.6) and (3.7). Then, by proceeding as in Lemma 3.2 we have that $p=\rho_{\mathrm{F}} \phi$. Thus by applying (3.8) we obtain

$$
\|\nabla g\|_{H^{s}\left(\Omega_{\mathrm{F}}\right)^{n}}=\frac{1}{\lambda}\|\boldsymbol{\nabla} p\|_{H^{s}\left(\Omega_{\mathrm{F}}\right)^{n}} \leq C\|\boldsymbol{\nabla} \phi\|_{H^{s}\left(\Omega_{\mathrm{F}}\right)^{n}} \leq C\|(\mathbf{f}, g)\| \leq C|(\mathbf{f}, g)| .
$$

\section{Finite element Discretization}

Let $\left\{\mathcal{T}_{h}^{F}\right\}$ and $\left\{\mathcal{T}_{h}^{S}\right\}$ be two families of regular tetrahedral meshes of $\Omega_{\mathrm{F}}$ and $\Omega_{\mathrm{S}}$, respectively; $h$ denotes as usual the meshsize. The meshes do not need to coincide on their common interface $\Gamma_{\mathrm{I}}$, but we assume that the faces of those tetrahedra in $\mathcal{T}_{h}^{S}$, lying on the external boundary of $\Omega_{\mathrm{S}}$, are completely contained either on $\Gamma_{\mathrm{D}}$ or on $\Gamma_{\mathrm{N}}$.

In order to approximate the operator $\mathcal{M}$ we have to compute a solution of problem (2.20). For this we use standard piecewise linear finite elements. Let $\mathcal{L}_{h}\left(\Omega_{\mathrm{F}}\right)$ denote the space of continuous piecewise linear functions on $\mathcal{T}_{h}^{F}$ and let $\mathcal{W}_{h}:=\mathcal{L}_{h}\left(\Omega_{\mathrm{F}}\right) / \mathbb{R} \subset \mathcal{W}$. We define an approximate operator $\mathcal{M}_{h}: \mathcal{V} \longrightarrow \mathcal{W}_{h}$ by 
$\mathcal{M}_{h}(\mathbf{f}, g):=\phi_{h}$, with $\phi_{h}$ being the unique solution in $\mathcal{W}_{h}$ of the discrete Neumann problem

$$
\int_{\Omega_{\mathrm{F}}} \rho_{\mathrm{F}} \boldsymbol{\nabla} \phi_{h} \cdot \nabla \psi_{h}=\int_{\Omega_{\mathrm{F}}} \frac{1}{c^{2}} g \psi_{h}+\int_{\Gamma_{\mathrm{I}}} \rho_{\mathrm{F}} \mathbf{f} \cdot \boldsymbol{\nu} \psi_{h} \mathrm{~d} \Gamma \quad \forall \psi_{h} \in \mathcal{W}_{h}
$$

The error estimate for this approximation is very well known:

Lemma 4.1. For $(\mathbf{f}, g) \in \mathcal{V}$, let $\phi$ and $\phi_{h}$ be the solutions of problems (2.20) and (4.1), respectively. Then,

$$
\left\|\nabla\left(\phi-\phi_{h}\right)\right\|_{L^{2}\left(\Omega_{\mathrm{F}}\right)^{n}} \leq C h^{s}\|(\mathbf{f}, g)\|,
$$

where $s \in\left(\frac{1}{2}, 1\right]$ is such that estimate (3.8) is valid.

Proof. It is a direct consequence of standard finite element error estimates and Lemma 3.4

Now we define an approximation of $\mathbf{T}$. Let $\mathcal{L}_{h}\left(\Omega_{\mathrm{S}}\right)$ be the space of continuous piecewise linear functions on $\mathcal{T}_{h}^{S}$ and $\mathcal{Q}_{h}\left(\Omega_{\mathrm{F}}\right)$ the space of piecewise constant functions on $\mathcal{T}_{h}^{F}$. Let $\mathcal{X}_{h}:=\left\{\left(\mathbf{w}_{h}, q_{h}\right) \in \mathcal{L}_{h}\left(\Omega_{\mathrm{S}}\right)^{n} \times \mathcal{Q}_{h}\left(\Omega_{\mathrm{F}}\right):\left.\mathbf{w}_{h}\right|_{\Gamma_{\mathrm{D}}}=\mathbf{0}\right\}$ and $\mathcal{V}_{h}:=\mathcal{X}_{h} \cap \mathcal{V}$.

The following approximation property holds:

Lemma 4.2. There exists a linear operator $\mathbf{I}_{h}: \mathcal{V} \longrightarrow \mathcal{V}_{h}$ such that, if $p \in H^{1}\left(\Omega_{\mathrm{F}}\right)$ and $\mathbf{v} \in H^{1+t}\left(\Omega_{\mathrm{S}}\right)^{n}$ for some $t \in(0,1]$, then

$$
\left\|\mathbf{I}_{h}(\mathbf{v}, p)-(\mathbf{v}, p)\right\| \leq C h^{t}\left[\|\mathbf{v}\|_{H^{1+t}\left(\Omega_{\mathrm{S}}\right)^{n}}+\|p\|_{H^{1}\left(\Omega_{\mathrm{F}}\right)}\right] .
$$

Proof. Let $\mathbf{v}_{h}$ be the Clément interpolant of $\mathbf{v}$ in $\mathcal{L}_{h}\left(\Omega_{\mathrm{S}}\right)^{n}$ (see [7]). Let $\tilde{p}_{h}$ be the $L^{2}\left(\Omega_{\mathrm{F}}\right)$-orthogonal projection of $p$ onto the subspace $\mathcal{Q}_{h}\left(\Omega_{\mathrm{F}}\right)$. The standard error estimates yield

$$
\begin{aligned}
\left\|\mathbf{v}_{h}-\mathbf{v}\right\|_{H^{1}\left(\Omega_{\mathrm{S}}\right)^{n}} & \leq C h^{t}\|\mathbf{v}\|_{H^{1+t}\left(\Omega_{\mathrm{S}}\right)^{n}} \\
\left\|\tilde{p}_{h}-p\right\|_{L^{2}\left(\Omega_{\mathrm{F}}\right)} & \leq C h\|p\|_{H^{1}\left(\Omega_{\mathrm{F}}\right)} .
\end{aligned}
$$

Let $p_{h}:=\tilde{p}_{h}+d_{h}$, with $d_{h}$ a constant chosen such that

$$
\int_{\Omega_{\mathrm{F}}} \frac{1}{c^{2}} p_{h}+\int_{\Gamma_{\mathrm{I}}} \rho_{\mathrm{F}} \mathbf{v}_{h} \cdot \boldsymbol{\nu} \mathrm{d} \Gamma=0 .
$$

Then $\left(\mathbf{v}_{h}, p_{h}\right) \in \mathcal{V}_{h}$ and, since $\int_{\Omega_{\mathrm{F}}} \frac{1}{c^{2}} \tilde{p}_{h}=\int_{\Omega_{\mathrm{F}}} \frac{1}{c^{2}} p=-\int_{\Gamma_{\mathrm{I}}} \rho_{\mathrm{F}} \mathbf{v} \cdot \boldsymbol{\nu} \mathrm{d} \Gamma$, then

$$
d_{h}=-\frac{1}{\operatorname{meas}\left(\Omega_{\mathrm{F}}\right)}\left(\int_{\Omega_{\mathrm{F}}} \tilde{p}_{h}+\int_{\Gamma_{\mathrm{I}}} \rho_{\mathrm{F}} c^{2} \mathbf{v}_{h} \cdot \boldsymbol{\nu} \mathrm{d} \Gamma\right)=\frac{\rho_{\mathrm{F}} c^{2}}{\operatorname{meas}\left(\Omega_{\mathrm{F}}\right)} \int_{\Gamma_{\mathrm{I}}}\left(\mathbf{v}-\mathbf{v}_{h}\right) \cdot \boldsymbol{\nu} \mathrm{d} \Gamma .
$$

Hence, by using (4.3), we have

$$
\left|d_{h}\right| \leq C\left\|\mathbf{v}_{h}-\mathbf{v}\right\|_{H^{1}\left(\Omega_{\mathrm{S}}\right)^{n}} \leq C h^{t}\|\mathbf{v}\|_{H^{1+t}\left(\Omega_{\mathrm{S}}\right)^{n}} .
$$

Thus by defining $\mathbf{I}_{h}(\mathbf{v}, p):=\left(\mathbf{v}_{h}, p_{h}\right)$, the proof follows from (4.3), (4.4) and (4.5).

Now let $\mathbf{T}_{h}: \mathcal{V} \longrightarrow \mathcal{V}$ be the linear bounded operator given by $\mathbf{T}_{h}(\mathbf{f}, g)=$ $\left(\mathbf{v}_{h}, p_{h}\right)$, with $\left(\mathbf{v}_{h}, p_{h}\right) \in \mathcal{V}_{h}$ being the solution of the discretized source problem

$$
a\left(\left(\mathbf{v}_{h}, p_{h}\right),\left(\mathbf{w}_{h}, q_{h}\right)\right)=b_{h}\left((\mathbf{f}, g),\left(\mathbf{w}_{h}, q_{h}\right)\right) \quad \forall\left(\mathbf{w}_{h}, q_{h}\right) \in \mathcal{V}_{h},
$$


where $b_{h}: \mathcal{V} \times \mathcal{V} \longrightarrow \mathbb{R}$ is the bilinear form defined by

$$
b_{h}((\mathbf{v}, p),(\mathbf{w}, q)):=\int_{\Omega_{\mathrm{S}}} \rho_{\mathrm{S}} \mathbf{v} \cdot \mathbf{w}+\int_{\Omega_{\mathrm{F}}} \frac{1}{c^{2}} \mathcal{M}_{h}(\mathbf{v}, p) q+\int_{\Gamma_{\mathrm{I}}} \rho_{\mathrm{F}} \mathcal{M}_{h}(\mathbf{v}, p) \mathbf{w} \cdot \boldsymbol{\nu} \mathrm{d} \Gamma .
$$

Notice that (4.6) is a nonconforming approximation of (3.3), because $b$ has been replaced by $b_{h}$. The following lemma provides an estimate for the corresponding consistency term which will be used below:

Lemma 4.3. There exists a constant $C>0$ such that, for all $(\mathbf{f}, g) \in \mathcal{V}$ and $\left(\mathbf{w}_{h}, q_{h}\right) \in \mathcal{V}_{h}$,

$$
\left|b\left((\mathbf{f}, g),\left(\mathbf{w}_{h}, q_{h}\right)\right)-b_{h}\left((\mathbf{f}, g),\left(\mathbf{w}_{h}, q_{h}\right)\right)\right| \leq C h^{s}|(\mathbf{f}, g)|\left\|\left(\mathbf{w}_{h}, q_{h}\right)\right\|,
$$

where $s \in\left(\frac{1}{2}, 1\right]$ is such that estimate (3.8) holds. Furthermore, if $(\mathbf{f}, g)$ is an eigenfunction of $\mathbf{T}$, then

$$
\left|b\left((\mathbf{f}, g),\left(\mathbf{w}_{h}, q_{h}\right)\right)-b_{h}\left((\mathbf{f}, g),\left(\mathbf{w}_{h}, q_{h}\right)\right)\right| \leq C h^{2 s}|(\mathbf{f}, g)|\left\|\left(\mathbf{w}_{h}, q_{h}\right)\right\| .
$$

Proof. Let $\phi=\mathcal{M}(\mathbf{f}, g)$ and $\phi_{h}=\mathcal{M}_{h}(\mathbf{f}, g)$. Let $\psi=\mathcal{M}\left(\mathbf{w}_{h}, q_{h}\right)$ and $\psi_{h}=$ $\mathcal{M}_{h}\left(\mathbf{w}_{h}, q_{h}\right)$. Then, by applying (2.20), (4.1), (3.2) and (4.2), we have

$$
\begin{aligned}
b\left((\mathbf{f}, g),\left(\mathbf{w}_{h}, q_{h}\right)\right) & -b_{h}\left((\mathbf{f}, g),\left(\mathbf{w}_{h}, q_{h}\right)\right) \\
& =\int_{\Omega_{\mathrm{F}}} \frac{1}{c^{2}}\left(\phi-\phi_{h}\right) q_{h}+\int_{\Gamma_{\mathrm{I}}} \rho_{\mathrm{F}}\left(\phi-\phi_{h}\right) \mathbf{w}_{h} \cdot \boldsymbol{\nu} \mathrm{d} \Gamma \\
& =\int_{\Omega_{\mathrm{F}}} \rho_{\mathrm{F}} \boldsymbol{\nabla}\left(\phi-\phi_{h}\right) \cdot \boldsymbol{\nabla} \psi \\
& =\int_{\Omega_{\mathrm{F}}} \rho_{\mathrm{F}} \boldsymbol{\nabla}\left(\phi-\phi_{h}\right) \cdot \nabla\left(\psi-\psi_{h}\right) \\
& =\int_{\Omega_{\mathrm{F}}} \rho_{\mathrm{F}} \boldsymbol{\nabla} \phi \cdot \nabla\left(\psi-\psi_{h}\right) \\
& \leq C h^{s}|(\mathbf{f}, g)|\left\|\left(\mathbf{w}_{h}, q_{h}\right)\right\| .
\end{aligned}
$$

Assume now that $(\mathbf{f}, g)$ is an eigenfunction of $\mathbf{T}$. Then from Lemmas 4.1 and 3.5 we have

$$
\left\|\boldsymbol{\nabla}\left(\phi-\phi_{h}\right)\right\|_{L^{2}\left(\Omega_{\mathrm{F}}\right)^{n}} \leq C h^{s}\|(\mathbf{f}, g)\| \leq C h^{s}|(\mathbf{f}, g)| .
$$

Therefore, instead of (4.7) we have in this case

$$
\begin{aligned}
b\left((\mathbf{f}, g),\left(\mathbf{w}_{h}, q_{h}\right)\right)-b_{h}\left((\mathbf{f}, g),\left(\mathbf{w}_{h}, q_{h}\right)\right) & =\int_{\Omega_{\mathrm{F}}} \rho_{\mathrm{F}} \boldsymbol{\nabla}\left(\phi-\phi_{h}\right) \cdot \nabla\left(\psi-\psi_{h}\right) \\
& \leq C h^{2 s}|(\mathbf{f}, g)|\left\|\left(\mathbf{w}_{h}, q_{h}\right)\right\| .
\end{aligned}
$$

A proof similar to that of Lemma 3.1 shows that $b_{h}$ is symmetric and its restriction to $\mathcal{V}_{h} \times \mathcal{V}_{h}$ is positive definite. Thus the operator $\mathbf{T}_{h}: \mathcal{V}_{h} \longrightarrow \mathcal{V}_{h}$ turns out to be self-adjoint and positive definite with respect to $a$ and $b_{h}$. Hence all of its eigenvalues are real and positive.

The following lemma implies uniform convergence of $\mathbf{T}_{h}$ to $\mathbf{T}$ as $h$ goes to 0 , which will allow us to use the spectral approximation theory in [1]: 
Lemma 4.4. There exists a constant $C>0$ such that, for $(\mathbf{f}, g) \in \mathcal{V}$,

$$
\frac{\left\|\left(\mathbf{T}-\mathbf{T}_{h}\right)(\mathbf{f}, g)\right\|}{|(\mathbf{f}, g)|} \leq C h^{r}
$$

with $r=\min \{s, t\}$, where $t \in(0,1]$ and $s \in\left(\frac{1}{2}, 1\right]$ are such that (3.8) and (3.4) hold. Furthermore, if $(\mathbf{f}, g)$ is an eigenfunction of $\mathbf{T}$, then the previous inequality holds for $r=t$.

Proof. This is a direct consequence of the First Strang's Lemma (see, for instance, [6]) and Lemmas 4.2, [3.2 and 4.3. In the case of $(\mathbf{f}, g)$ being an eigenfunction of $\mathbf{T}$, $r=\min \{2 s, t\}=t$.

As a consequence of the lemma above, since the bilinear form $b$ defining the norm $|\cdot|$ is continuous on $\mathcal{V}$, then $\mathbf{T}_{h}$ converge to $\mathbf{T}$ in norm $\|\cdot\|$ (actually also in $|\cdot|$ ) and hence isolated parts of the spectrum of $\mathbf{T}$ are approximated by isolated parts of the spectrum of $\mathbf{T}_{h}$ (see [11]). More precisely, for any eigenvalue $\lambda$ of $\mathbf{T}$ of finite multiplicity $m$, there exist exactly $m$ eigenvalues $\lambda_{h}^{(1)}, \ldots, \lambda_{h}^{(m)}$ of $\mathbf{T}_{h}$ (repeated according to their respective multiplicities) converging to $\lambda$ as $h$ goes to zero. Furthermore, no spurious modes can arise as is typical in some other discretizations of spectral problems in fluid-structure interaction (see, for instance, 10]).

From now on and until the end of this section let $\lambda$ be a positive fixed eigenvalue of $\mathbf{T}$ of finite multiplicity $m$ and let $\mathbf{E}$ be its associated eigenspace. For $h$ small enough, let $\lambda_{h}^{(1)}, \ldots, \lambda_{h}^{(m)}$ be the $m$ eigenvalues of $\mathbf{T}_{h}$ converging to $\lambda$ and let $\mathbf{E}_{h}$ be the direct sum of the corresponding eigenspaces. Thus, by applying the spectral approximation theory for compact operators as stated in [1] (Theorem 7.1) and by using Lemma 4.4, we obtain the following error estimates:

Theorem 4.5. There exists a constant $C>0$, independent of $h$, such that

1. for each $\left(\mathbf{v}_{h}, p_{h}\right) \in \mathbf{E}_{h}$, dist $\left(\left(\mathbf{v}_{h}, p_{h}\right), \mathbf{E}\right) \leq C h^{t}\left\|\left(\mathbf{v}_{h}, p_{h}\right)\right\|$,

2. for each $(\mathbf{v}, p) \in \mathbf{E}$, dist $\left((\mathbf{v}, p), \mathbf{E}_{h}\right) \leq C h^{t}\|(\mathbf{v}, p)\|$,

where dist denotes the distance in norm $\|\cdot\|$ and $t \in(0,1]$ is such that estimate (3.4) holds.

Finally, regarding the eigenvalues, we are going to prove a theorem providing an improved order of convergence. For this purpose we will exploit the fact that our spectral problem is "variationally formulated" in the sense of [1] (Section 8). However the results in this reference cannot be directly applied to our case since (4.6) is a nonconforming approximation of (3.3).

Let us denote by $\mathcal{H}$ the Hilbert space obtained as the completion of the space $\mathcal{V}$ with respect to the norm $|\cdot|$. Then $(\mathcal{V},\|\cdot\|)$ is continuously and densely included in $(\mathcal{H},|\cdot|)$. Thus the operator $\mathbf{T}$ can be uniquely extended to $\mathcal{H}$ and this extension is also self-adjoint with respect to $b$. Similarly, $\mathbf{T}_{h}$ can also be extended to $\mathcal{H}$; however this extension is not self-adjoint with respect to $b$. Indeed, let us call $\mathbf{T}_{h}^{*}$ its adjoint with respect to this inner product; then, $\forall(\mathbf{v}, p),(\mathbf{w}, q) \in \mathcal{H}$,

$$
b\left(\mathbf{T}_{h}^{*}(\mathbf{v}, p),(\mathbf{w}, q)\right)=b\left((\mathbf{v}, p), \mathbf{T}_{h}(\mathbf{w}, q)\right)=a\left(\mathbf{T}(\mathbf{v}, p), \mathbf{T}_{h}(\mathbf{w}, q)\right),
$$

whereas

$$
b\left(\mathbf{T}_{h}(\mathbf{v}, p),(\mathbf{w}, q)\right)=a\left(\mathbf{T}_{h}(\mathbf{v}, p), \mathbf{T}(\mathbf{w}, q)\right),
$$


which, in general, do not coincide. Nevertheless a larger order of convergence can be proved for the approximation of the eigenvalues:

Theorem 4.6. There exists a strictly positive constant $C$, independent of $h$, such that

$$
\left|\lambda-\lambda_{h}^{(i)}\right| \leq C h^{2 r}, \quad i=1, \ldots, m,
$$

with $r:=\min \{s, t\}$, where $t \in(0,1]$ and $s \in\left(\frac{1}{2}, 1\right]$ are such that (3.4) and (3.8) hold.

Proof. By specializing Theorem 7.3 of [1] to our situation we have that, for $i=$ $1, \ldots, m$,

$$
\begin{array}{r}
\left|\lambda-\lambda_{h}^{(i)}\right| \leq C\left[\sup _{\substack{(\mathbf{f}, g) \in \mathbf{E} \\
|(\mathbf{f}, g)|=1}} \sup _{\substack{(\widehat{\mathbf{f}}, \widehat{g}) \in \mathbf{E} \\
|(\hat{\mathbf{f}}, \widehat{g})|=1}}\left|b\left(\left(\mathbf{T}-\mathbf{T}_{h}\right)(\mathbf{f}, g),(\widehat{\mathbf{f}}, \widehat{g})\right)\right|\right. \\
\left.\quad+\left.\left|\left(\mathbf{T}-\mathbf{T}_{h}\right)\right|_{\mathbf{E}}||\left(\mathbf{T}-\mathbf{T}_{h}^{*}\right)\right|_{\mathbf{E}} \mid\right] .
\end{array}
$$

Lemma 4.4 provides a bound of $\left|\left(\mathbf{T}-\mathbf{T}_{h}\right)\right|_{\mathbf{E}} \mid$, so we only need to estimate the two remaining terms in the expression above. For the first one, let $(\mathbf{f}, g),(\widehat{\mathbf{f}}, \widehat{g}) \in \mathbf{E}$ and let

$$
\begin{array}{ll}
(\mathbf{v}, p):=\mathbf{T}(\mathbf{f}, g) \in \mathcal{V}, & (\widehat{\mathbf{v}}, \widehat{p}):=\mathbf{T}(\widehat{\mathbf{f}}, \widehat{g}) \in \mathcal{V} \\
\left(\mathbf{v}_{h}, p_{h}\right):=\mathbf{T}_{h}(\mathbf{f}, g) \in \mathcal{V}_{h}, & \left(\widehat{\mathbf{v}}_{h}, \widehat{p}_{h}\right):=\mathbf{T}_{h}(\widehat{\mathbf{f}}, \widehat{g}) \in \mathcal{V}_{h}
\end{array}
$$

Then

$$
\begin{aligned}
b\left(\left(\mathbf{T}-\mathbf{T}_{h}\right)(\mathbf{f}, g),(\widehat{\mathbf{f}}, \widehat{g})\right)= & a\left((\mathbf{v}, p)-\left(\mathbf{v}_{h}, p_{h}\right),(\widehat{\mathbf{v}}, \widehat{p})\right) \\
= & a\left(\left(\mathbf{v}-\mathbf{v}_{h}, p-p_{h}\right),\left(\widehat{\mathbf{v}}-\widehat{\mathbf{v}}_{h}, \widehat{p}-\widehat{p}_{h}\right)\right) \\
& +\left[b\left((\mathbf{f}, g),\left(\widehat{\mathbf{v}}_{h}, \widehat{p}_{h}\right)\right)-b_{h}\left((\mathbf{f}, g),\left(\widehat{\mathbf{v}}_{h}, \widehat{p}_{h}\right)\right)\right] .
\end{aligned}
$$

The first term in the r.h.s of this expression can be easily bounded by using the continuity of $a$ and again Lemma 4.4.

$$
\left|a\left(\left(\mathbf{v}-\mathbf{v}_{h}, p-p_{h}\right),\left(\widehat{\mathbf{v}}-\widehat{\mathbf{v}}_{h}, \widehat{p}-\widehat{p}_{h}\right)\right)\right| \leq C h^{2 t}|(\mathbf{f}, g)||(\widehat{\mathbf{f}}, \widehat{g})|,
$$

whereas for the second one we use Lemma 4.3 and the uniform boundedness of $\mathbf{T}_{h}: \mathcal{H} \longrightarrow \mathcal{V}_{h}:$

$$
\begin{aligned}
\left|b\left((\mathbf{f}, g),\left(\widehat{\mathbf{v}}_{h}, \widehat{p}_{h}\right)\right)-b_{h}\left((\mathbf{f}, g),\left(\widehat{\mathbf{v}}_{h}, \widehat{p}_{h}\right)\right)\right| & \leq C h^{2 s}|(\mathbf{f}, g)|\left\|\left(\widehat{\mathbf{v}}_{h}, \widehat{p}_{h}\right)\right\| \\
& \leq C h^{2 s}|(\mathbf{f}, g)||(\widehat{\mathbf{f}}, \widehat{g})| .
\end{aligned}
$$

Thus, as a consequence of (4.8), (4.9) and (4.10), we obtain

$$
\sup _{\substack{(\mathbf{f}, g) \in \mathbf{E} \\|(\mathbf{f}, g)|=1}} \sup _{\substack{(\widehat{\mathbf{f}}, \widehat{g}) \in \mathbf{E} \\|(\hat{\mathbf{f}}, \widehat{g})|=1}}\left|b\left(\left(\mathbf{T}-\mathbf{T}_{h}\right)(\mathbf{f}, g),(\widehat{\mathbf{f}}, \widehat{g})\right)\right| \leq C h^{\min \{2 s, 2 t\}} .
$$


On the other hand, regarding $\left|\left(\mathbf{T}-\mathbf{T}_{h}^{*}\right)\right|_{\mathbf{E}} \mid$, since $\mathcal{V}$ is dense in $\mathcal{H}$, we have

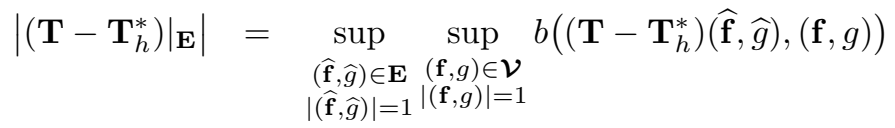

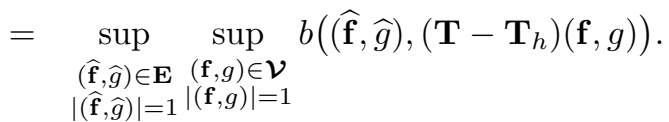

Then $b\left((\widehat{\mathbf{f}}, \widehat{g}),\left(\mathbf{T}-\mathbf{T}_{h}\right)(\mathbf{f}, g)\right)=b\left(\left(\mathbf{T}-\mathbf{T}_{h}\right)(\mathbf{f}, g),(\widehat{\mathbf{f}}, \widehat{g})\right)$ can be handled as above. However, now $(\mathbf{f}, g) \notin \mathbf{E}$; so, by repeating the arguments above, we only obtain

$$
\left|b\left(\left(\mathbf{T}-\mathbf{T}_{h}\right)(\mathbf{f}, g),(\widehat{\mathbf{f}}, \widehat{g})\right)\right| \leq C h^{\min \{s, 2 t\}}|(\mathbf{f}, g)||(\widehat{\mathbf{f}}, \widehat{g})| .
$$

Nevertheless, since Lemma 4.4 yields $\left|\left(\mathbf{T}-\mathbf{T}_{h}\right)\right|_{\mathbf{E}} \mid \leq C h^{t}$, this is enough to conclude the theorem.

\section{Computer implementation}

In the previous section it was proved that the spectrum and eigenfunctions of $\mathbf{T}_{h}$ converge to those of $\mathbf{T}$ as $h$ goes to zero. Equivalently, the solutions of the spectral problem (2.18)-(2.19) are approximated by those of the following discrete eigenvalue problem:

Find $\omega_{h} \geq 0$ and $\mathbf{0} \neq\left(\mathbf{v}_{h}, p_{h}\right) \in \mathcal{V}_{h}$ such that

$$
\begin{aligned}
\int_{\Omega_{\mathrm{S}}} \boldsymbol{\sigma}\left(\mathbf{v}_{h}\right): \varepsilon\left(\mathbf{w}_{h}\right)+\int_{\Omega_{\mathrm{F}}} \frac{1}{\rho_{\mathrm{F}} c^{2}} p_{h} q_{h} \\
=\omega_{h}^{2}\left[\int_{\Omega_{\mathrm{S}}} \rho_{\mathrm{S}} \mathbf{v}_{h} \cdot \mathbf{w}_{h}+\int_{\Omega_{\mathrm{F}}} \frac{1}{c^{2}} \dot{\phi}_{h} q_{h}+\int_{\Gamma_{\mathrm{I}}} \rho_{\mathrm{F}} \dot{\phi}_{h} \mathbf{w}_{h} \cdot \boldsymbol{\nu} \mathrm{d} \Gamma\right] \\
\forall\left(\mathbf{w}_{h}, q_{h}\right) \in \mathcal{V},
\end{aligned}
$$

with $\dot{\phi}_{h} \in \mathcal{W}_{h}$ such that

$$
\int_{\Omega_{\mathrm{F}}} \rho_{\mathrm{F}} \boldsymbol{\nabla} \dot{\phi}_{h} \cdot \nabla \dot{\psi}_{h}=\int_{\Omega_{\mathrm{F}}} \frac{1}{c^{2}} p_{h} \dot{\psi}_{h}+\int_{\Gamma_{\mathrm{I}}} \rho_{\mathrm{F}} \mathbf{v}_{h} \cdot \boldsymbol{\nu} \dot{\psi}_{h} \mathrm{~d} \Gamma \quad \forall \dot{\psi}_{h} \in \mathcal{W}_{h}
$$

In the equations above dotted variables (e.g., $\dot{\phi}_{h}, \dot{\psi}_{h}$ ) are used to denote classes of the quotient space $\mathcal{W}_{h}=\mathcal{L}_{h}\left(\Omega_{\mathrm{F}}\right) / \mathbb{R}$. We will use this notation throughout this section to distinguish these classes from their own members, which will be denoted by the corresponding undotted symbol (e.g., $\phi_{h}, \psi_{h}$, respectively).

From the computational point of view it would be convenient to avoid imposing the constraint in the definition of $\mathcal{V}_{h}$. To this goal, consider the following discrete spectral problem, which is formally obtained by adding equation (5.2) multiplied by $\omega_{h}^{2}$ to equation (5.1), and using a member of $\dot{\phi}_{h}$ such that (5.1) is valid for any test function in $\mathcal{X}_{h}$ : 
Find $\omega_{h} \geq 0$ and $\mathbf{0} \neq\left(\mathbf{v}_{h}, p_{h}, \phi_{h}\right) \in \mathcal{X}_{h} \times \mathcal{L}_{h}\left(\Omega_{\mathrm{F}}\right)$ such that

$$
\begin{aligned}
& \int_{\Omega_{\mathrm{S}}} \boldsymbol{\sigma}\left(\mathbf{v}_{h}\right): \boldsymbol{\varepsilon}\left(\mathbf{w}_{h}\right)+\int_{\Omega_{\mathrm{F}}} \frac{1}{\rho_{\mathrm{F}} c^{2}} p_{h} q_{h} \\
&=\omega_{h}^{2}\left[\int_{\Omega_{\mathrm{S}}} \rho_{\mathrm{S}} \mathbf{v}_{h} \cdot \mathbf{w}_{h}+\int_{\Omega_{\mathrm{F}}} \frac{1}{c^{2}} \phi_{h} q_{h}+\int_{\Gamma_{\mathrm{I}}} \rho_{\mathrm{F}} \phi_{h} \mathbf{w}_{h} \cdot \boldsymbol{\nu} \mathrm{d} \Gamma\right. \\
&\left.+\int_{\Omega_{\mathrm{F}}} \frac{1}{c^{2}} p_{h} \psi_{h}+\int_{\Gamma_{\mathrm{I}}} \rho_{\mathrm{F}} \mathbf{v}_{h} \cdot \boldsymbol{\nu} \psi_{h} \mathrm{~d} \Gamma-\int_{\Omega_{\mathrm{F}}} \rho_{\mathrm{F}} \boldsymbol{\nabla} \phi_{h} \cdot \nabla \psi_{h}\right] \\
& \forall\left(\mathbf{w}_{h}, q_{h}, \psi_{h}\right) \in \mathcal{X}_{h} \times \mathcal{L}_{h}\left(\Omega_{\mathrm{F}}\right) .
\end{aligned}
$$

As shown below, this is a well-posed algebraic generalized eigenvalue problem with symmetric sparse matrices. Clearly it has $\omega_{h}^{2}=0$ as an eigenvalue with associated eigenspace $\{\boldsymbol{0}\} \times\{0\} \times \mathcal{L}_{h}\left(\Omega_{\mathrm{F}}\right)$. In the following lemma we show that, apart from $\omega_{h}^{2}=0$, problems (5.1) $-(5.2)$ and (5.3) are equivalent. More precisely, the following result holds:

Lemma 5.1. Let $\omega_{h}>0$. Then, $\omega_{h}, \mathbf{0} \neq\left(\mathbf{v}_{h}, p_{h}\right) \in \mathcal{V}_{h}$ and $\dot{\phi}_{h} \in \mathcal{W}_{h}$ are solution of (5.1) -(5.2) if and only if $\omega_{h}$ and $\mathbf{0} \neq\left(\mathbf{v}_{h}, p_{h}, \phi_{h}\right) \in \mathcal{X}_{h} \times \mathcal{L}_{h}\left(\Omega_{\mathrm{F}}\right)$ are solutions of (5.3), with $\phi_{h}$ being the member of $\dot{\phi}_{h}$ satisfying

$$
\int_{\Omega_{\mathrm{F}}} \frac{1}{\rho_{\mathrm{F}} c^{2}} p_{h}=\omega_{h}^{2} \int_{\Omega_{\mathrm{F}}} \frac{1}{c^{2}} \phi_{h}
$$

Proof. Let $\omega_{h}>0, \mathbf{0} \neq\left(\mathbf{v}_{h}, p_{h}\right) \in \mathcal{V}_{h}$ and $\dot{\phi} \in \mathcal{W}_{h}$ be solutions of (5.1)-(5.2). Let $\phi_{h}$ be the member of $\dot{\phi}_{h}$ satisfying (5.4). Then, by using $\phi_{h}$ instead of $\dot{\phi}_{h}$ in (5.1), this equation is satisfied for $\left(\mathbf{w}_{h}, q_{h}\right)=(\mathbf{0}, 1)$ too, and thus it is valid for all $\left(\mathbf{w}_{h}, q_{h}\right) \in \boldsymbol{X}_{h}=\mathcal{V}_{h} \oplus\langle\{(\mathbf{0}, 1)\}\rangle$. On the other hand, since $\left(\mathbf{v}_{h}, p_{h}\right) \in \mathcal{V}_{h}$, (5.2) is valid for all $\psi_{h} \in \mathcal{L}_{h}\left(\Omega_{\mathrm{F}}\right)$. Therefore $\omega_{h}$ and $\mathbf{0} \neq\left(\mathbf{v}_{h}, p_{h}, \phi_{h}\right) \in \mathcal{X}_{h} \times \mathcal{L}_{h}\left(\Omega_{\mathrm{F}}\right)$ are solutions of (5.3).

Conversely, let $\omega_{h}>0$ and $\mathbf{0} \neq\left(\mathbf{v}_{h}, p_{h}, \phi_{h}\right) \in \mathcal{X}_{h} \times \mathcal{L}_{h}\left(\Omega_{\mathrm{F}}\right)$ be solutions of (5.3). By testing this equation with $\left(\mathbf{w}_{h}, q_{h}, \psi_{h}\right)=(\mathbf{0}, 0,1)$ we prove that $\left(\mathbf{v}_{h}, p_{h}\right) \in \mathcal{V}_{h}$. Hence (5.2) is well-posed for $\dot{\psi} \in \mathcal{W}_{h}$ and satisfied by $\dot{\phi}_{h}$. On the other hand we prove that (5.1) holds by using $\left(\mathbf{w}_{h}, q_{h}, 0\right)$, with $\left(\mathbf{w}_{h}, q_{h}\right) \in \mathcal{V}_{h}$, as a test function in (5.3). Therefore $\omega_{h}, \mathbf{0} \neq\left(\mathbf{v}_{h}, p_{h}\right) \in \mathcal{V}_{h}$ and $\dot{\phi} \in \mathcal{W}_{h}$ are solutions of (5.1)-(5.2).

Finally we will show that the discrete spectral problem (5.3) leads to a well-posed algebraic generalized eigenvalue problem with symmetric sparse matrices. Let us write it in matrix form. Let $\Upsilon, \Phi, \Pi, \Xi, \Psi$ and $\Theta$ denote the vectors of nodal components of $\mathbf{v}_{h}, \phi_{h}, p_{h}, \mathbf{w}_{h}, \psi_{h}$ and $q_{h}$, respectively. The matrices associated with the bilinear forms in the variational formulation (5.3) are defined by

$$
\begin{array}{rlrl}
\Xi^{\mathrm{t}} \mathbf{K} \Upsilon & =\int_{\Omega_{\mathrm{S}}} \boldsymbol{\sigma}\left(\mathbf{v}_{h}\right): \boldsymbol{\varepsilon}\left(\mathbf{w}_{h}\right), & \Xi^{\mathrm{t}} \mathbf{M} \Upsilon & =\int_{\Omega_{\mathrm{S}}} \rho_{\mathrm{S}} \mathbf{v}_{h} \cdot \mathbf{w}_{h}, \\
\Psi^{\mathrm{t}} \mathbf{F} \Phi & =\int_{\Omega_{\mathrm{F}}}^{\rho_{\mathrm{F}} \boldsymbol{\nabla} \phi_{h} \cdot \nabla \psi_{h},} & \Theta^{\mathrm{t}} \mathbf{D} \Pi=\int_{\Omega_{\mathrm{F}}} \frac{1}{\rho_{\mathrm{F}} c^{2}} p_{h} q_{h}, \\
\Psi^{\mathrm{t}} \mathbf{B} \Pi=\int_{\Omega_{\mathrm{F}}} \frac{1}{c^{2}} p_{h} \psi_{h}, & \Psi^{\mathrm{t}} \mathbf{C} \Upsilon & =\int_{\Gamma_{\mathrm{I}}} \rho_{\mathrm{F}} \mathbf{v}_{h} \cdot \boldsymbol{\nu} \psi_{h} \mathrm{~d} \Gamma .
\end{array}
$$

$\mathbf{K}$ and $\mathbf{M}$ are the standard stiffness and mass matrices of the solid, respectively. Similarly, $\mathbf{D}$ and $\mathbf{F}$ are the stiffness and mass matrices corresponding to the fluid. 
On the other hand, $\mathbf{C}$ is a matrix for the interface coupling between solid displacement and fluid displacement potential, while $\mathbf{B}$ is a coupling matrix between the latter and the fluid pressure.

Problem (5.3) is written in terms of these matrices in the following way:

$$
\left(\begin{array}{ccc}
\mathbf{K} & \mathbf{0} & \mathbf{0} \\
\mathbf{0} & \mathbf{D} & \mathbf{0} \\
\mathbf{0} & \mathbf{0} & \mathbf{0}
\end{array}\right)\left(\begin{array}{c}
\Upsilon \\
\Pi \\
\Phi
\end{array}\right)=\omega_{h}^{2}\left(\begin{array}{ccc}
\mathbf{M} & \mathbf{0} & \mathbf{C}^{\mathrm{t}} \\
\mathbf{0} & \mathbf{0} & \mathbf{B}^{\mathrm{t}} \\
\mathbf{C} & \mathbf{B} & -\mathbf{F}
\end{array}\right)\left(\begin{array}{c}
\Upsilon \\
\Pi \\
\Phi
\end{array}\right)
$$

This is a well-posed generalized eigenvalue problem with symmetric indefinite sparse matrices. As was said above, $\omega_{h}^{2}=0$ is an eigenvalue of this problem with eigenspace $\{\mathbf{0}\} \times\{0\} \times \mathcal{L}_{h}\left(\Omega_{\mathrm{F}}\right)$.

Apart from $\omega_{h}^{2}=0$ it has a finite number of eigenvalues, which are exactly those of Problem (5.1) with $\dot{\phi}_{h}=\mathcal{M}_{h}\left(\mathbf{v}_{h}, p_{h}\right)$. Since this last problem involves symmetric positive definite matrices, the number of nonzero eigenvalues is equal to the dimension of $\mathcal{V}_{h}$ (and all of them are strictly positive).

This implies that for any value of $\sigma$, different from all these eigenvalues, the matrix

$$
\left(\begin{array}{ccc}
\mathbf{K} & \mathbf{0} & \mathbf{0} \\
\mathbf{0} & \mathbf{D} & \mathbf{0} \\
\mathbf{0} & \mathbf{0} & \mathbf{0}
\end{array}\right)-\sigma\left(\begin{array}{ccc}
\mathbf{M} & \mathbf{0} & \mathbf{C}^{\mathrm{t}} \\
\mathbf{0} & \mathbf{0} & \mathbf{B}^{\mathrm{t}} \\
\mathbf{C} & \mathbf{B} & -\mathbf{F}
\end{array}\right)=\left(\begin{array}{ccc}
\mathbf{K}-\sigma \mathbf{M} & \mathbf{0} & -\sigma \mathbf{C}^{\mathrm{t}} \\
\mathbf{0} & \mathbf{D} & -\sigma \mathbf{B}^{\mathrm{t}} \\
-\sigma \mathbf{C} & -\sigma \mathbf{B} & \sigma \mathbf{F}
\end{array}\right)
$$

is non singular and sparse. Consequently any "shift and invert" method could be conveniently used to solve this eigenproblem.

On the other hand, since the pressure field is approximated by piecewise constant functions, matrix $D$ is diagonal. Because of this, the degrees of freedom corresponding to the pressure can be statically condensed in the "invert" step of any "shift and invert" method, without destroying the sparseness of the matrices (see 12 for details). Therefore, the dimension of the system which should be effectively solved is equal to the number of degrees of freedom of the solid displacement plus those of the fluid potential, i.e., the same number as if the nonsymmetric displacement/potential formulation in [17] were used.

In reference 12 a convenient two-step algorithm based on this static condensation is introduced to solve the eigenvalue problem (5.5). Further implementation issues are discussed in this reference and 2D numerical experiments exhibiting the good performance of the method are also reported.

\section{REFERENCES}

1. I. Babuška and J. Osborn, Eigenvalue problems, in Handbook of Numerical Analysis, Vol. II, P.G. Ciarlet and J.L. Lions, eds., North Holland, Amsterdam, 1991. CMP 91:14

2. A. Bermúdez, R. Durán, M.A. Muschietti, R. Rodríguez and J. Solomin, Finite element vibration analysis of fluid-solid systems without spurious modes, SIAM J. Numer. Anal., 32 (1995) 1280-1295. MR 96e:73072

3. A. Bermúdez, R. Durán and R. Rodríguez, Finite element analysis of compressible and incompressible fluid-solid systems, Math. Comp., 67 (1998) 111-136. MR 98c:73073

4. A. Bermúdez, L. Hervella-Nieto and R. Rodríguez, Finite element computation of three dimensional elastoacoustic vibrations, J. Sound \& Vibr., 219 (1999) 277-304.

5. A. Bermúdez and R. Rodríguez, Finite element computation of the vibration modes of a fluid-solid system, Comp. Methods Appl. Mech. Eng., 119 (1994) 355-370. MR 95j:73064

6. P.G. Ciarlet, Basic error estimates for elliptic problems, in Handbook of Numerical Analysis, Vol. II, P.G. Ciarlet and J.L. Lions, eds., North Holland, Amsterdam, 1991. CMP 91:14

7. P. Clément, Approximation by finite element functions using local regularization, RAIRO Anal. Numér., 9 (1975) 77-84. MR 53:4569 
8. M. Dauge, Problèmes de Neumann et de Dirichlet sur un polyèdre dans $\mathbb{R}^{3}$ : régularité dans des spaces de Sobolev $L_{p}$, C. R. Acad. Sci. Paris, Série I, 307 (1988) 27-32. MR 90a:35057

9. M. Dauge, Elliptic boundary value problems on corner domains: smoothness and asymptotics of solutions, Lecture Notes in Mathematics 1341, Springer, Berlin, 1988. MR 91a:35078

10. M. Hamdi, Y. Ousset and G. Verchery, A displacement method for the analysis of vibrations of coupled fluid-structure systems, Internat. J. Numer. Methods Eng., 13 (1978) 139-150.

11. T. Kato, Perturbation theory for linear operators, Springer, Berlin, 1976. MR 53:11389

12. M. Mellado and R. Rodríguez, Efficient solution of fluid-structure vibration problems, Appl. Numer. Math. 36 (2001) 389-400. CMP 2001:10

13. H. Morand and R. Ohayon, Substructure variational analysis of the vibrations of coupled fluid-structure systems. Finite element results, Internat. J. Numer. Methods Eng., 14, (1979) 741-755.

14. H.J-P. Morand and R. Ohayon, Fluid-structure interactions, John Wiley \& Sons, New York, 1995.

15. T. von Petersdorff, Boundary value problems of elasticity in polyhedra: singularities and approximation with boundary element methods, PhD Thesis, Technical University Darmstadt, Darmstadt, Germany, 1989

16. T. von Petersdorff and E.P. Stephan, Regularity of mixed boundary value problems in $\mathbb{R}^{3}$ and boundary integral methods on graded meshes, Math. Methods Appl. Sci., 12 (1990) 229-249. MR 91k:35049

17. O.C. Zienkiewicz and R.L. Taylor, The finite element method, Mc Graw Hill, London, 1989.

Departamento de Matemática Aplicada, Universidade de Santiago de Compostela, 15706 Santiago de Compostela, Spain

E-mail address: mabermud@usc.es

Departamento de Ingeniería Matemática, Universidad de Concepción, Casilla 160-C, Concepción, Chile

E-mail address: rodolfo@ing-mat.udec.cl 